\title{
Candida endocarditis with mycotic pulmonary emboli following re-do Rastelli operation
}

\author{
N T Wijesekera, M N Sheppard, M J Mullen
}

Heart 2004;90:e34 (http://www.heartjnl.com/cgi/content/full/90/6/e34). doi: 10.1136/hrt.2004.034736

A case of a 19 year old patient with Candida endocarditis complicated by pulmonary infarction and pulmonary mycotic abscesses following replacement of a right ventricle to pulmonary artery homograft conduit is presented. Despite preceding hospital admissions with probable septic pulmonary emboli, diagnosis was made only after massive pulmonary haemorrhage that ultimately proved fatal. This case highlights that Candida endocarditis should be considered in patients with symptoms and signs compatible with bacterial endocarditis when blood cultures are negative, especially in the setting of congenital cardiac malformations, and illustrates the high mortality associated with delayed diagnosis.

$\mathrm{R}$ ight heart infective endocarditis is a rare clinical entity with an insidious presentation. Fungal endocarditis is similarly uncommon, and is mainly seen in congenital heart disease, especially after open heart surgery. Candida species is the most frequently implicated fungal organism. Here we describe a unique case of Candida endocarditis complicating replacement of a right ventricle to pulmonary artery homograft conduit, where the diagnosis was delayed despite multiple hospital admissions with probable septic pulmonary emboli.

\section{CASE REPORT}

A 19 year old male had been diagnosed in early infancy with complete transposition of the great arteries, ventricular septal defect, and subpulmonary stenosis. At 7 days old he had a left Blalock-Taussig (BT) shunt, and at 2 years old he required a right BT shunt and atrial septectomy. A Rastelli procedure was performed at age 8 years. At 18 years old, he underwent conduit replacement using a $23 \mathrm{~mm}$ valved homograft because of conduit stenosis; there were no immediate complications.

Between 3-6 months after re-do surgery, he presented three times to his local hospital with pleuritic chest pains and dyspnoea. On the first and second occasions, he was diagnosed with culture negative lower respiratory tract infections; both episodes were treated with empirical courses of broad spectrum antibiotics which resulted in symptomatic improvement. On the third occasion, a ventilation perfusion scan suggested left lower lobe pulmonary embolus and he was started on warfarin. Transthoracic echocardiography showed no evidence of infective endocarditis.

Ten months after surgery, he was again admitted with similar symptoms including an intermittent cough with pink expectoration. He also reported notable postoperative weight loss. He was treated with cefuroxime and clarithromycin for presumed recurrent pneumonia and his condition appeared to improve. However, his relapsing clinical course raised the suspicion of underlying infective endocarditis, so he was transferred to a tertiary centre for further management.

On presentation to the Royal Brompton Hospital, the patient was pale and cachetic but he was afebrile and there were no peripheral stigmata of endocarditis. Examination revealed a blood pressure of 91/62 $\mathrm{mm} \mathrm{Hg}$, pulse of 76 beats/ min, and respirations of 20 breaths/min. Jugular venous pressure was low. Cardiac auscultation detected an ejection systolic murmur and a new holodiastolic murmur loudest in the pulmonary area. He had clinical and radiographic evidence of right and left lower lobe consolidation. There was moderate splenomegaly. Admission blood tests showed a microcytic anaemia (haemoglobin $9.2 \mathrm{~g} / \mathrm{dl}$, mean corpuscular volume $76 \mathrm{fl}$ ), and mildly elevated white cell count $\left(10.1 \times 10^{9} / 1\right)$ and inflammatory markers (C-reactive protein $52 \mathrm{mg} / \mathrm{l})$.

While awaiting further investigations, his condition abruptly deteriorated with massive haemoptysis $(500 \mathrm{ml}$ in 10 minutes) resulting in profound hypoxia and haemodynamic compromise. He was resuscitated and transferred to the intensive care unit (ICU). Flexible bronchoscopy found bleeding from the right middle bronchus. Chest computed tomography revealed a right lower lobe pulmonary artery aneurysm with thrombus and surrounding haemorrhagic infarct (fig 1). Transoesophageal echocardiography demonstrated vegetations on a thickened and severely regurgitant conduit valve. Serial sputum and blood cultures had been sterile. Nevertheless, he was treated empirically with teicoplanin, ceftazidime, and gentamicin. Persistent bleeding necessitated de-clotting of the airways via rigid bronchoscopy, but it was felt that he would not tolerate surgical

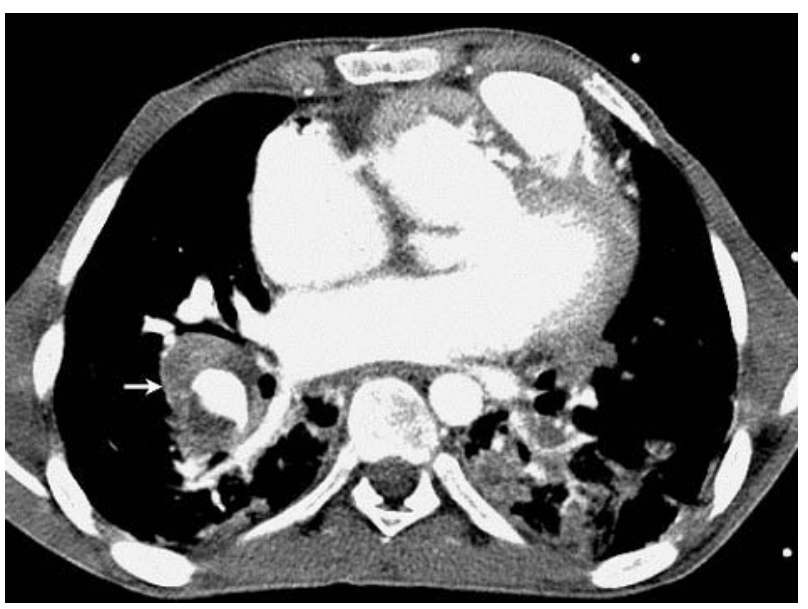

Figure 1 Contrast enhanced computed tomography of chest showing extensive consolidation in both lower lobes representing either infarct or infection. The rounded area of consolidation in the right lower lobe is suggestive of thrombus in the right pulmonary artery with surrounding haemorrhagic infarct (arrowed). 

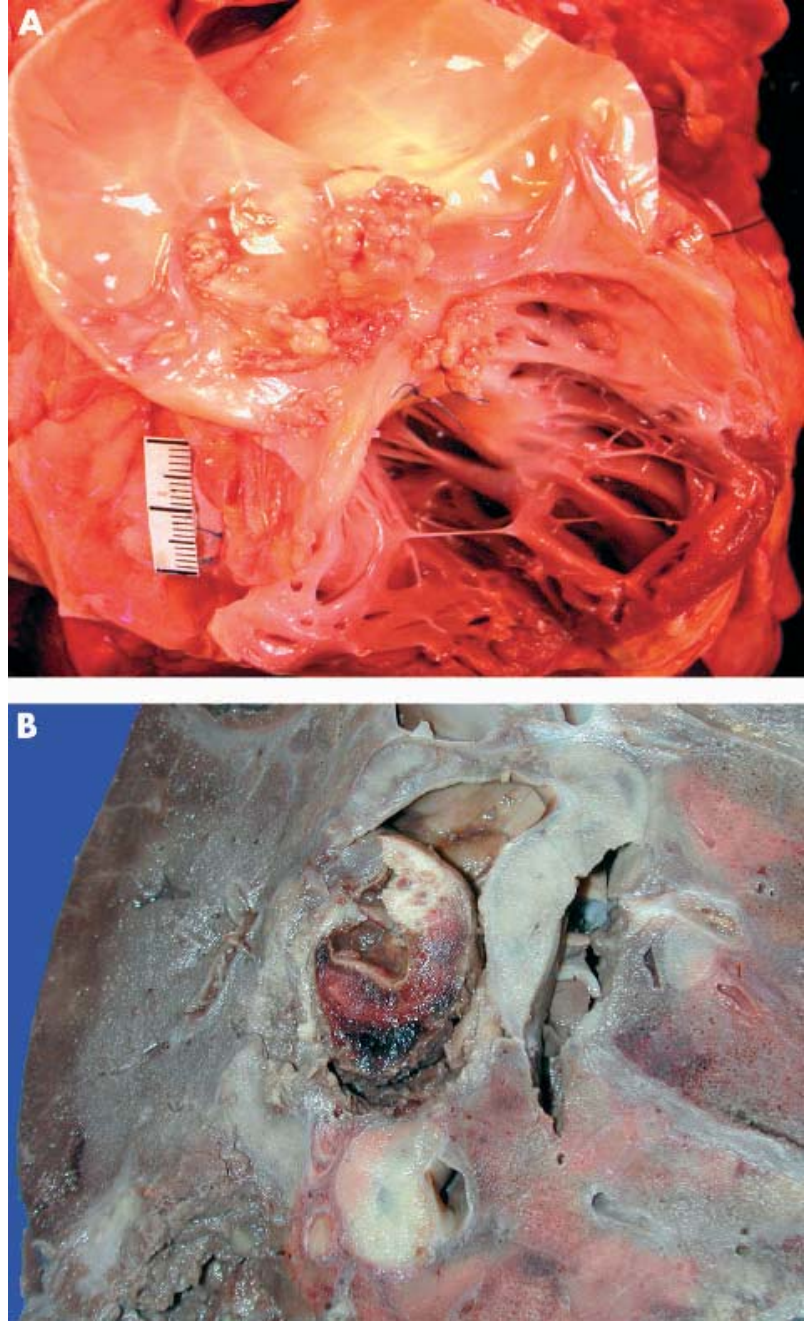

Figure 2 Macroscopic views. Panel A shows vegetation adherent to the sinus and remnant of the posterior semi-lunar leaflet of the homograft conduit. Panel B shows aneursymal dilatation of the right lower lobe pulmonary artery with necrotic organised thrombus attached to the artery wall. The surrounding lung parenchyma has areas of consolidation and abscess formation adjacent to the artery aneurysm.

resection of infected/infarcted tissue. After six days in the ICU, Candida albicans was cultured in blood and bronchoalveolar lavage. Liposomal amphotericin B was commenced. However, he continued to deteriorate despite active therapy and, after eight days in the ICU, he died.

Postmortem examination of the homograft conduit revealed a large vegetation adherent to the posterior semilunar valve causing perforation of the leaflet (fig 2A). The lungs were heavy and congested, with aneurysmal dilatation of both lower lobe pulmonary arteries, the right more prominent than the left, with necrotic organised thrombus attached to the walls of the arteries (fig 2B). Sections of the conduit valve and pulmonary aneurysms stained for fungal organisms showed evidence of Candida. The lung parenchyma had extensive abscess formation and foci of infarction in both lower lobes.

\section{DISCUSSION}

Infective endocarditis is a rare complication following the Rastelli operation, with an approximate lifetime incidence of $5 \%$ (site and organism unspecified). ${ }^{2}$ Fungal endovascular infection involving the conduit homograft valve in these patients has not previously been reported.

The clinical manifestations of right heart endocarditis commonly relate to the respiratory system as pulmonary embolisation of vegetations from the infected valve. Cardiac signs may be absent or minimal in the early course of the disease. ${ }^{4}$ It is likely that the postoperative "chest infections" in the above patient represented septic embolic phenomena.

Candida endocarditis may complicate a wide variety of cardiac procedures but is most frequent after valvar surgery. Intravascular access or prophylactic antibiotics given in the postoperative period may increase susceptibility to infection, although, as in our case, a clear predisposing factor may not be apparent. The risk of fungal endocarditis caused by extrinsic valve contamination following allograft valve replacement is approximately $0.3 \% .^{5}$ Most cases of Candida related valvitis occur in the first two postoperative months, but presentation may occur later. Embolisation to major vessels is a distinctive characteristic of the organism. ${ }^{6}$ This case highlights that Candida endocarditis should be considered in any patient with symptoms and signs compatible with bacterial endocarditis when blood cultures are negative.

Fungal endocarditis is associated with a particularly high mortality. The optimal management of the Candida endocarditis is to initiate amphotericin B treatment and to surgically resect infected tissue as soon as possible. A previously reported patient with truncus arteriosus communis had an isolated large Candida vegetation within the prosthetic pulmonary valve that was successfully treated in this manner. ${ }^{7}$ However, the above case underscores the difficulty in diagnosing the lesion, and illustrates the high mortality associated with delayed diagnosis.

\section{Authors' affiliations}

N T Wijesekera, M J Mullen, Department of Cardiology, Royal

Brompton Hospital, Sydney Street, London

M N Sheppard, Department of Histopathology, Royal Brompton Hospital

Correspondence to: Dr N Wijesekera, Department of Cardiology, Royal Brompton Hospital, Sydney Street, London, SW3 6NP, UK ;

n.wijesekera@ic.ac.uk

Accepted 16 February 2004

\section{REFERENCES}

1 Rubinstein E, Lang R. Fungal endocarditis. Eur Heart J 1995;16:84-9.

2 Dearani JA, Danielson GK, Puga FJ, et al. Late results of the Rastelli operation for transposition of the great arteries. Semin Thorac Cardiovasc Surg Pediatr Card Surg Annu 2003;4:3-15.

3 Kreutzer C, De Vive J, Oppido G. Twenty-five-year experience with Rastelli repair for transposition of the great arteries. J Thorac Cardiovasc Surg 2002; 123:192-3.

4 Tolan $M$, Clarke S, Schofield $P$, et al. Homograft replacement of fungal endocarditic pulmonary valve. Eur J Cardiothoracic Surg 1995;9:528-30.

5 Gall KM, Smith S, Wilmette C, et al. Allograft heart valve sterilization: a sixyear in-depth analysis of a 25 year experience with low dose antibiotics. J Thorac Cardiovasc Surg 1995;110:680-7.

6 Collins GJ Jr, Rich NM, Hobson RW, et al. Multiple mycotic aneurysms due to Candida endocarditis. Ann Surg 1977;186:136-9.

7 Hausser M, Hess J, Belohradsky BH. Treatment of Candida albicans endocarditis: Case report and a review. Infection 2003;31:125-7. 